\title{
Active and fast charge-state switching of single NV centres in diamond by in-plane Al-Schottky junctions
}

\author{
Christoph Schreyvogel ${ }^{* 1}$, Vladimir Polyakov ${ }^{1}$, Sina Burk ${ }^{2}$, Helmut Fedder $^{2}$, \\ Andrej Denisenko ${ }^{2}$, Felipe Fávaro de Oliveira ${ }^{2}$, Ralf Wunderlich ${ }^{3}$, Jan Meijer ${ }^{3}$, \\ Verena Zuerbig $^{1}$, Jörg Wrachtrup ${ }^{2,4}$ and Christoph E. Nebel ${ }^{1}$
}

\section{Letter}

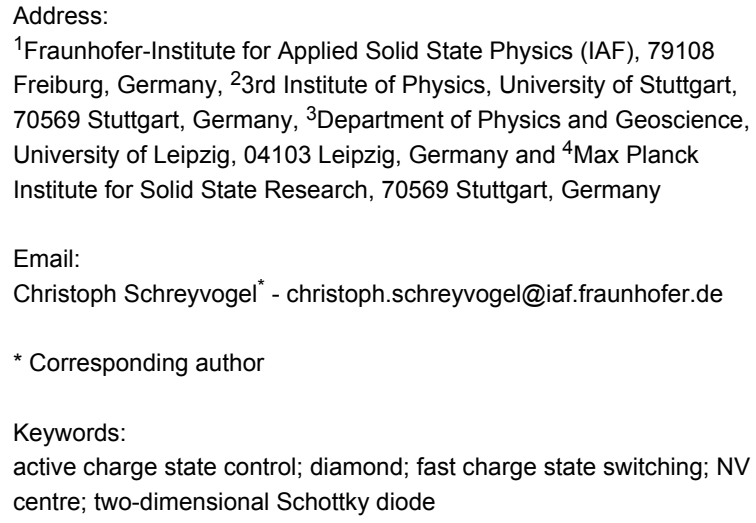

Beilstein J. Nanotechnol. 2016, 7, 1727-1735. doi:10.3762/bjnano.7.165

Received: 25 July 2016

Accepted: 31 October 2016

Published: 16 November 2016

This article is part of the Thematic Series "Functional nanostructures optical and magnetic properties".

Guest Editor: P. Leiderer

(C) 2016 Schreyvogel et al.; licensee Beilstein-Institut. License and terms: see end of document.

\begin{abstract}
In this paper, we demonstrate an active and fast control of the charge state and hence of the optical and electronic properties of single and near-surface nitrogen-vacancy centres (NV centres) in diamond. This active manipulation is achieved by using a twodimensional Schottky-diode structure from diamond, i.e., by using aluminium as Schottky contact on a hydrogen terminated diamond surface. By changing the applied potential on the Schottky contact, we are able to actively switch single NV centres between all three charge states $\mathrm{NV}^{+}, \mathrm{NV}^{0}$ and $\mathrm{NV}^{-}$on a timescale of 10 to $100 \mathrm{~ns}$, corresponding to a switching frequency of 10-100 MHz. This switching frequency is much higher than the hyperfine interaction frequency between an electron spin (of $\mathrm{NV}^{-}$) and a nuclear spin (of ${ }^{15} \mathrm{~N}$ or ${ }^{13} \mathrm{C}$ for example) of $2.66 \mathrm{kHz}$. This high-frequency charge state switching with a planar diode structure would open the door for many quantum optical applications such as a quantum computer with single NVs for quantum information processing as well as single ${ }^{13} \mathrm{C}$ atoms for long-lifetime storage of quantum information. Furthermore, a control of spectral emission properties of single NVs as a single photon emitters - embedded in photonic structures for example - can be realized which would be vital for quantum communication and cryptography.
\end{abstract}

\section{Introduction}

The nitrogen-vacancy centre (NV centre) in diamond is known to exist in at least three different charge states $\left(\mathrm{NV}^{-}, \mathrm{NV}^{0}\right.$ and $\mathrm{NV}^{+}$state). Compared to the positive and the neutral charge state, the negative state $\left(\mathrm{NV}^{-}\right)$shows superior properties for electron-spin related applications in quantum physics, namely a perfect photostability [1], an optical initialization and read-out of its ground state electron spin [2] and an optically detected magnetic resonance [3] at ambient conditions. Due to these out- 
standing properties it can be used for applications such as single-spin magnetometry [4], imaging in life science [5], quantum computing and quantum cryptography $[6,7]$ at room temperature, i.e., without the need for cryogenic conditions.

For many quantum applications, near-surface $\mathrm{NV}^{-}$centres are required in order to efficiently couple out the emitted photoluminescence or to increase its sensitivity to external magnetic fields. However, one drawback is that near-surface NV centres are strongly affected by surface defects, surface terminations and adsorbates. Hence, its charge state switches in an uncontrolled way between $\mathrm{NV}^{-}, \mathrm{NV}^{0}$ and presumably $\mathrm{NV}^{+}$[8-10].

In addition, for realizing quantum computing applications such as quantum information processing with the electron spin of the $\mathrm{NV}^{-}$centre and quantum information storage with the nuclear spin of the ${ }^{15} \mathrm{~N}$ or ${ }^{13} \mathrm{C}$ atom, it is necessary not only to stabilize the $\mathrm{NV}^{-}$state but also to switch actively between $\mathrm{NV}^{-}$(bright state, with electron spin) and $\mathrm{NV}^{+}$(dark state, without electron spin). It was recently shown that a transfer of the spin state from an electron spin to a nuclear spin leads to very large $T_{2}$ times [11] which is required for quantum information storage as well as for conserving the entanglement with high fidelity [12] Therefore, an important requirement for realizing quantum optical applications with single NV centres is not only an active charge state control but also a fast switching between all three charge states $\mathrm{NV}^{-}, \mathrm{NV}^{0}$ and $\mathrm{NV}^{+}$.

To understand the importance of fast switching, we will now consider one application example of a quantum computer consisting of $\mathrm{NV}$ centres and ${ }^{13} \mathrm{C}$ atoms in the diamond crystal, arranged as quantum registers. The electron spin of an $\mathrm{NV}^{-}$ centre can be used for initialising, manipulating and processing of quantum information as well as entanglement of its spin state with other NVs as quantum bits. The nuclear spin of a ${ }^{13} \mathrm{C}$ atom can be used for storage of quantum information with a long lifetime. In order to achieve a long lifetime of stored quantum information with high fidelity we have - after transfer of the quantum state from the electron to the nuclear spin - to decouple the electron spin from the nuclear spin. For this, the $\mathrm{NV}$ centre need to be switched from $\mathrm{NV}^{-}$(optically active, with electron spin) to $\mathrm{NV}^{+}$(optically inactive, without electron spin). The stored quantum information can be read out optically by transferring the spin state from nuclear spin back to the electron spin. For this, the NV centre need to be switched back from $\mathrm{NV}^{+}$(optically inactive, without electron spin) to $\mathrm{NV}^{-}$(optically active, with electron spin). In both cases the switching speed should be faster than the rate of hyperfine interaction between an electron and nuclear spin of $\mathrm{A}_{/ /}=2.66 \mathrm{kHz}$ [13] to avoid any negative interplay between both spins during switching processes.
In order to control and to switch actively the charge state of a single NV centre, we have to control the position of the Fermilevel relative to charge transition levels $\mathrm{NV}^{+/ 0}$ and $\mathrm{NV}^{0 /-}$ of the centre within the bandgap of diamond. The charge transition level of a defect centre is defined as a level at which the centre takes up or loses an electron when the Fermi level crosses this level. A control of the Fermi-level position can be performed either passively by the chemical control via surface termination with oxygen [8-10] or fluorine [14] or actively by an electrical control with structures such as a solution-gated field effect transistor [15], a pin-diode [16] or an in-plane gate transistor [17]. However, the above-mentioned passive control is static, i.e., the charge state cannot be switched on demand. In addition, with the above-mentioned techniques of electrical control it enables a control and switching between only the two (optical active) charge states $\mathrm{NV}^{-}$and $\mathrm{NV}^{0}$.

In this work, we demonstrate for the first time an active control and a fast switching of the charge state of single NV centres between all three charge states $\left(\mathrm{NV}^{-}, \mathrm{NV}^{0}\right.$ and $\left.\mathrm{NV}^{+}\right)$by using a two-dimensional diamond Schottky-diode structure on a hydrogen-terminated (H-terminated) diamond surface. A H-terminated diamond surface exhibits a two-dimensional hole channel a few nanometres below the surface and an aluminium (Al) contact forms a two-dimensional Schottky junction (i.e., in planar configuration) with the p-type surface conductive channel inducing a lateral hole depletion layer below and next to the $\mathrm{Al}$ contact $[18,19]$. By applying potentials on the Al-Schottky contact, the applied electric field in the depletion region of the Schottky junction induces a band bending modulation which shifts the Fermi-level over NV charge transition level and the charge state of this centre is switched in an active and controlled way $[20,21]$.

This paper is structured as follows: First as an introduction, we give a very short summary of the fabrication of the Schottky diode and the results published in a previous paper showing that spectral properties of the emitted photoluminescence of single NV centres is changed upon applying reverse bias potentials on the Al-Schottky contact indicating an active switching from the nonfluorescent $\mathrm{NV}^{+}$state to the fluorescent state $\mathrm{NV}^{0}$ or $\mathrm{NV}^{-}$ and vice versa [21]. Then we will show the latest results on the fast switching of the NV charge state. Details on the diamond growth, fabrication of Schottky-diode and experimental setup for optical detection are described in [20,21].

\section{Sample preparation}

An Ib (100) $3 \times 3 \times 0.3 \mathrm{~mm}^{3}$ diamond plate from Element Six Ltd. was used as a substrate and a $300 \mu \mathrm{m}$ thick intrinsic epilayer with high purity (i.e., without optically active defect centres such as NV centres) was grown homoepitaxially onto 
the substrate using an ellipsoidal shaped microwave plasma-enhanced chemical vapour deposition (MWPECVD) reactor [22]. After growth, the substrate was removed by laser cutting, the epi-layer mechanically polished to get a smooth surface [23] and cleaned wet chemically which results in oxygen-termination (O-termination) of the surface.

In the next step, the freestanding intrinsic diamond sample was implanted with nitrogen ions with implantation energy of $5 \mathrm{keV}$ corresponding to a mean depth of $8.2 \mathrm{~nm}$ according to a SRIM (stopping and range of ions in matter) simulation [24]. The implanted dose of nitrogen was $10^{8}$ ions $/ \mathrm{cm}^{2}$ covering homogenously the surface. The sample was annealed for $2 \mathrm{~h}$ at $800{ }^{\circ} \mathrm{C}$ in vacuum to create $\mathrm{NV}$ centres. The formation efficiency is about $1-10 \%[25,26]$ which leads to an NV centre sheet density in of $[\mathrm{NV}]=10^{6}-10^{7} \mathrm{~cm}^{-2}$.

We used confocal microscopy with an excitation laser of $520 \mathrm{~nm}$ to detect the NV centre photoluminescence in the depletion region of the Schottky junction. Distinct spectral character- istics can be used to identify the charge state: the $\mathrm{NV}^{+}$centre does not show fluorescence upon optical excitation, whereas $\mathrm{NV}^{0}$ and $\mathrm{NV}^{-}$show a zero phonon line at $575 \mathrm{~nm}$ at $637 \mathrm{~nm}$, respectively, with their corresponding phonon side bands [8].

A PL-intensity mapping performed on the surface shows bright spots originating from NV luminescence (Figure 1a). An analysing of PL-mapping images of mapping size of $20 \times 20 \mu \mathrm{m}^{2}$ each reveals an average NV density of approximately $10^{7} \mathrm{~cm}^{-2}$. This would correspond to a formation efficiency of approximately $10 \%$ which is in agreement with formation efficiency values reported in literature $[25,26]$. The bright spots in the mapping images were identified as single $\mathrm{NV}^{-}$centres according to a measurement of the spectrum (Figure 1b) and the second-order photon autocorrelation with a Hanbury-Brown and Twiss interferometer setup [1,27,28], which shows a clear antibunching dip at $\tau=0 \mathrm{~s}$ (Figure 1c).

Before the realisation of a two-dimensional Schottky diode, the diamond surface was hydrogen-terminated (H-terminated) by (a)

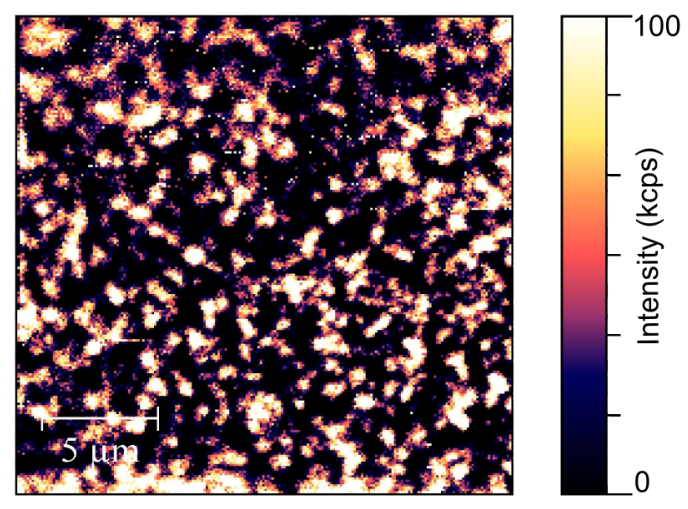

(c)

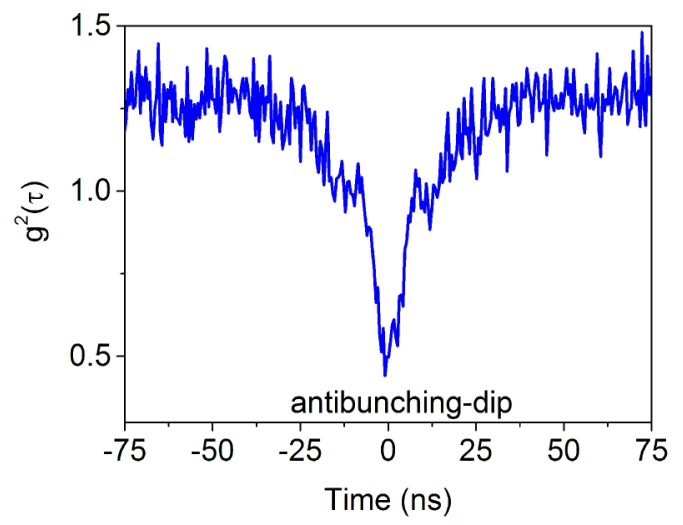

(b)

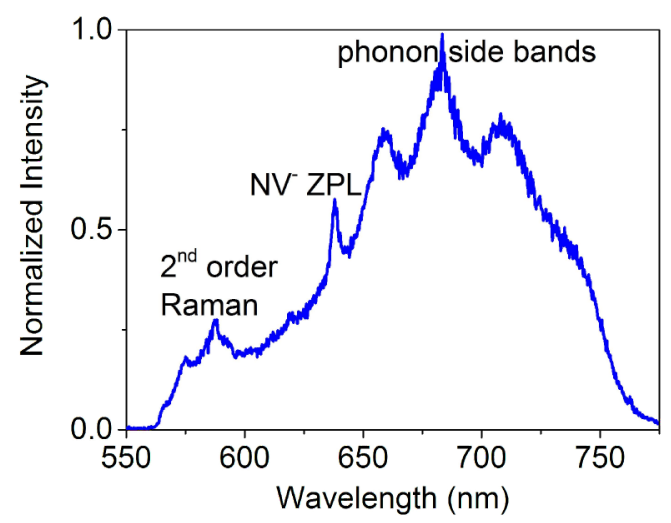

(d)

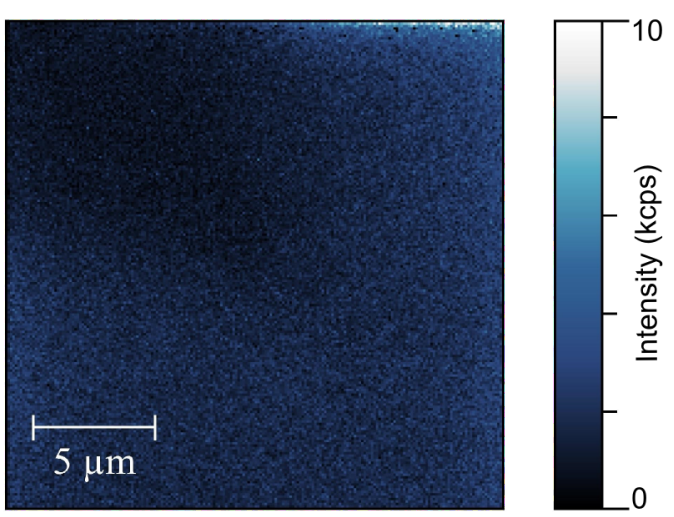

Figure 1: Formation and quenching of NV centres. (a) PL-intensity mapping performed on the diamond surface which shows bright spots. These bright spots are single $\mathrm{NV}^{-}$centres according to the corresponding (b) PL-spectrum and (c) second-order photon autocorrelation measurement performed with a Hanbury-Brown and Twiss interferometer setup showing a clear antibunching dip indicating single photon emission. (d) After $\mathrm{H}$-termination of the diamond surface the NV-emission is quenched due to the conversion from the fluorescent state $\mathrm{NV}^{-}$to $\mathrm{NV}^{+}$, i.e., only a PL-background of the surface is visible. 
applying a pure H-plasma in the MWPECVD reactor which results in the formation of a two-dimensional hole accumulation layer a few nanometres below the surface [18]. Due to the interaction with the two-dimensional hole channel, NV centres are quenched, i.e., switched to the nonfluorescent state $\mathrm{NV}^{+}$as proved by PL-intensity mappings which show no bright spots (Figure 1d).

For fabricating a two-dimensional Schottky diode structure, aluminium (Al) and gold ( $\mathrm{Au}$ ) contacts with a thickness of $200 \mathrm{~nm}$ each were deposited onto the diamond surface using photolithography with subsequent thermal evaporation of the metals. $\mathrm{Al}$ is a Schottky contact showing a barrier height of $570 \mathrm{meV}$ and $\mathrm{Au}$ is an Ohmic contact [18]. The contacts exhibit dimensions of $1 \mathrm{~mm} \times 300 \mu \mathrm{m}$ and were separated from each other by $400 \mu \mathrm{m}$. In order to have a defined conductive channel between the $\mathrm{Al}$ and $\mathrm{Au}$ contacts, the channel region including the contacts were protected with a photoresist and then the whole diamond surface surrounding the channel area were O-terminated via exposition to oxygen plasma. After lift-off process of the photoresist, the fabrication of the in-plane diamond Schottky diode is finished, which is shown schematically in Figure 2a. The current-voltage characteristic of this diode, measured at ambient conditions, shows very good Schottky properties (Figure 2b).

\section{Results and Discussion}

\section{Active charge-state switching}

As already published elsewhere, via analyzing PL-spectra at different potentials applied on the Schottky contact, we could demonstrate an active control of the charge state of a single NV centre in the depletion region of the in-plane Schottky junction [21]. At zero bias there is only a PL-background of the diamond surface (black spectrum in Figure 3), since the NV centre is in the $\mathrm{NV}^{+}$state which shows no fluorescence. Upon applying a reverse bias voltage of $+15 \mathrm{~V}$, the measured spectrum clearly shows $\mathrm{NV}^{0}$ emission with the characteristic zero phonon line at $575 \mathrm{~nm}$ and its phonon side band (red spectrum in Figure 3). By increasing the reverse bias voltage to $+20 \mathrm{~V}$, the measured spectrum shows $\mathrm{NV}^{-}$emission with the characteristic zero phonon line at $637 \mathrm{~nm}$ and its phonon side band (blue spectrum in Figure 3). After switching off the applied bias voltage, the recorded PL-spectrum revealed only the previously measured background spectrum of the diamond surface (black spectrum of Figure 3), which indicates the NV centre to be in the nonfluorescent $\mathrm{NV}^{+}$state.

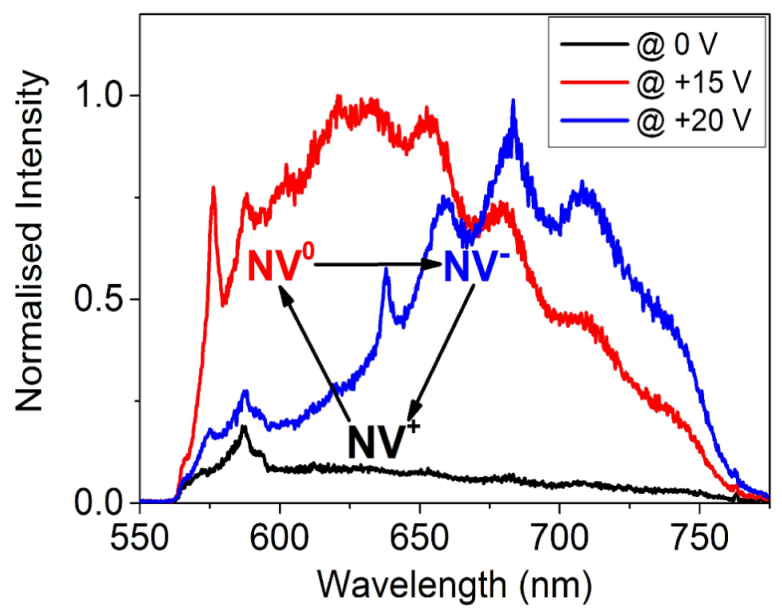

Figure 3: Active charge state control of a single NV centre. At zero bias there is only a PL-background of the diamond surface (black spectrum) since the $\mathrm{NV}$ centre is in the nonfluorescent $\mathrm{NV}^{+}$state due to the interaction with the two-dimensional hole accumulation layer. Upon applying a reverse bias potential of $+15 \mathrm{~V}$, we detect a spectrum of $\mathrm{NV}^{0}$ emission (red spectrum). Upon applying a higher reverse bias potential $(+20 \mathrm{~V})$ we detect a spectrum of $\mathrm{NV}^{-}$emission (blue spectrum) (a)

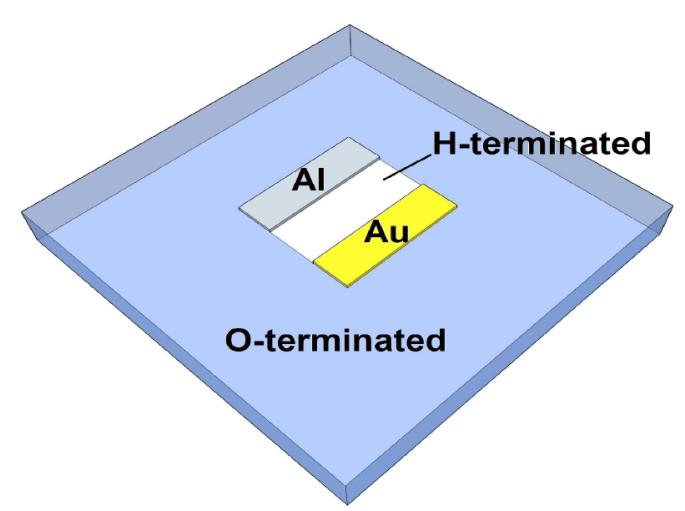

(b)

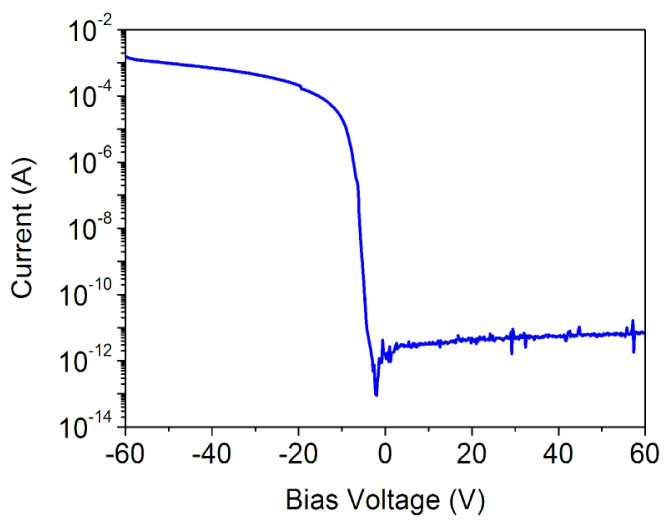

Figure 2: In-plane Schottky diode from diamond. (a) Schematic figure of an in-plane Al Schottky diode on an H-terminated diamond surface. (b) The current-voltage properties of the fabricated in-plane Al-Schottky diode measured at ambient conditions. 


\section{Fast charge-state switching}

In order to answer the question of how fast the charge state of a single NV centre can be switched with the two-dimensional diamond Schottky diode and to understand the underlying switching mechanisms, we performed a time-resolved measurement of NV-intensity during switching between the fluorescent $\left(\mathrm{NV}^{-}\right.$and $\left.\mathrm{NV}^{0}\right)$ and nonfluorescent $\left(\mathrm{NV}^{+}\right)$states. To record the time evolution of the $\mathrm{NV}$ intensity during switching between $\mathrm{NV}^{-}$or $\mathrm{NV}^{0}$ and $\mathrm{NV}^{+}$, the single $\mathrm{NV}$ centre was illuminated continuously with a $520 \mathrm{~nm}$ laser and the emitted NV-photoluminescence was routed via a beam splitter to two avalanche photo diodes (APD) with corresponding $\mathrm{NV}\left(\mathrm{NV}^{0}\right.$ and $\left.\mathrm{NV}^{-}\right)$ filters in front of them. The time resolution for detecting the intensity change by an avalanche photo diode is in the range of $1-10 \mathrm{~ns}$. A schematic of this measurement setup is shown in Figure 4.

For switching the applied potential on the Schottky diode, we used a FPGA-based pulse generator in combination with an amplifier. For switching between $\mathrm{NV}^{0} \leftrightarrow \mathrm{NV}^{+}$or $\mathrm{NV}^{-} \leftrightarrow \mathrm{NV}^{+}$, a rectangle pulse of amplitude $\pm 15 \mathrm{~V}$ or $\pm 20 \mathrm{~V}$ with a period of $4 \mu$ s was applied. The voltage ramping for switching between $\pm 20 \mathrm{~V}$ takes place within $100 \mathrm{~ns}$. Thus, a switching from $\mathrm{NV}^{-}$ $(+20 \mathrm{~V})$ to $\mathrm{NV}^{+}(<+15 \mathrm{~V})$ and vice versa will take place theoretically within $10-15 \mathrm{~ns}$.

The result of the time-resolved $\mathrm{NV}$-intensity measurement in Figure 5 shows that switching from $\mathrm{NV}^{-}$to $\mathrm{NV}^{+}$generally takes place much faster than switching from $\mathrm{NV}^{+}$to $\mathrm{NV}^{-}$. In Figure 6, all results of a time-resolved intensity measurement for switching between the states $\mathrm{NV}^{-} \leftrightarrow \mathrm{NV}^{+}$or $\mathrm{NV}^{0} \leftrightarrow \mathrm{NV}^{+}$ are summarised.

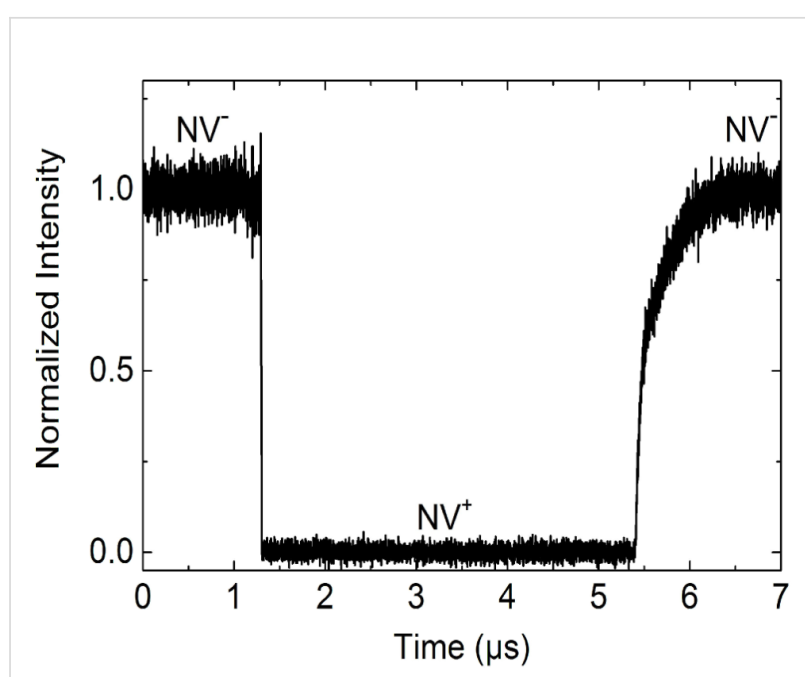

Figure 5: Fast charge state switching. The result of the time-resolved $\mathrm{NV}$-intensity measurement shows that switching from $\mathrm{NV}^{-}$to $\mathrm{NV}^{+}$ takes place much faster than switching from $\mathrm{NV}^{+}$to $\mathrm{NV}^{-}$. Since in this case we used an $\mathrm{NV}^{-}$filter in front of an ADP, the maximum and the minimum of the detected intensity corresponds to the $\mathrm{NV}^{-}$and $\mathrm{NV}^{+}$ state respectively.

From fitting the following exponential function to the decrease and increase of the NV-intensity, we could deduce the time constants $\tau_{1}$ and $\tau_{2}$ :

$$
y=y_{0}+A_{1} \cdot e^{-\left(x-x_{0}\right) / \tau_{1}}+A_{2} \cdot e^{-\left(x-x_{0}\right) / \tau_{2}} .
$$

A summary of measured time constants for switching a single $\mathrm{NV}$ centre between the fluorescent and nonfluorescent states as well as the corresponding calculated maximum (theoretical) switching frequencies is given in Table 1. According to the

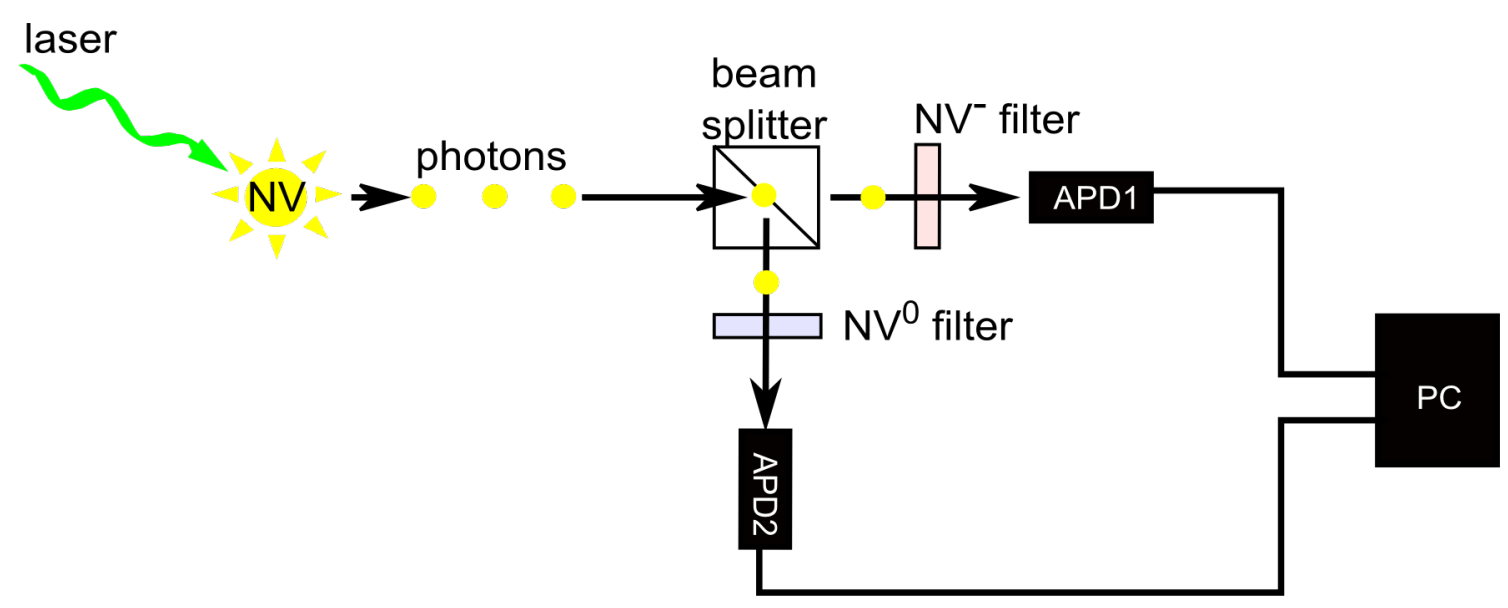

Figure 4: Schematic setup for time-resolved measurement of NV-intensity. To record the time evolution of the intensity of $\mathrm{NV}^{-}$and $\mathrm{NV}^{0}$ emission during switching between $\mathrm{NV}^{-}$or $\mathrm{NV}^{0}$ and $\mathrm{NV}^{+}$, the single $\mathrm{NV}$ centre was illuminated continuously with a $520 \mathrm{~nm}$ laser during the switching cycles and the emitted NV-photoluminescence was routed via a beam splitter to two avalanche photo diodes with corresponding NV filters in front of them. 
(a)

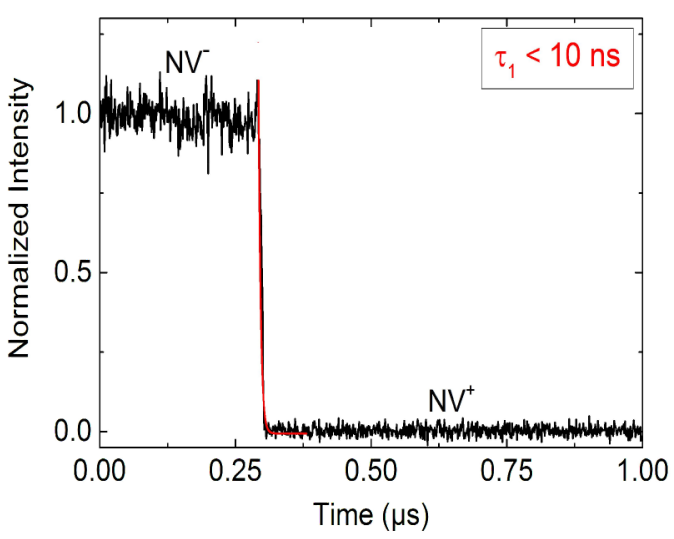

(c)

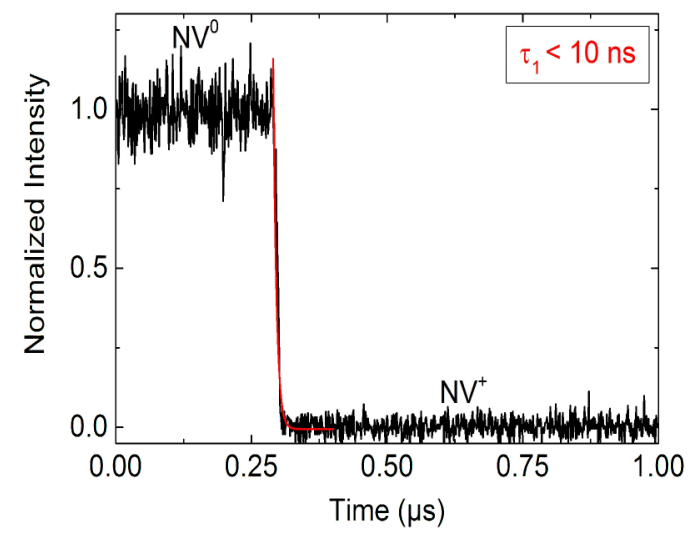

(b)

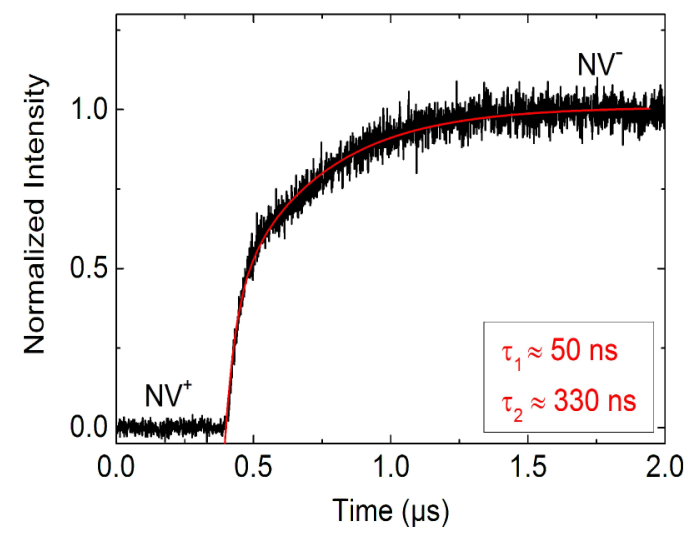

(d)

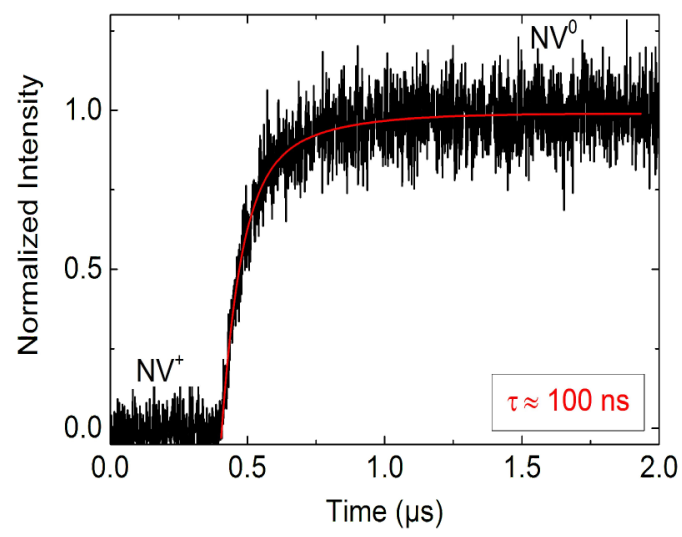

Figure 6: Time constant for charge state switching. Time constants deduced from fitting an exponential function to the decay and rise of NV-intensity for the transitions (a) $\mathrm{NV}^{-} \rightarrow \mathrm{NV}^{+}$(b) $\mathrm{NV}^{+} \rightarrow \mathrm{NV}^{-}$(c) $\mathrm{NV}^{0} \rightarrow \mathrm{NV}^{+}$as well as (d) $\mathrm{NV}^{+} \rightarrow \mathrm{NV}^{0}$. Since the time resolution of this measurement is in the range of 1-10 ns, the deduced time constants in (a) and (c) are upper limits. According to the measured time constants, a switching rate between $1 \mathrm{MHz}$ (charging NV) and $100 \mathrm{MHz}$ (discharging NV) seems to be possible. This is much faster than the rate of hyperfine interaction between an electron and nuclear spin of $A_{/ /}=2.66 \mathrm{kHz}$.

Table 1: Summary of measured switching speed. Measured time constants for switching a single NV centre between the fluorescent and non-fluorescent states as well as the corresponding calculated maximum (theoretical) switching rates.
NV-transition
time constant
max. switching frequency

$\begin{array}{lcc}\mathrm{NV}^{-} \rightarrow \mathrm{NV}^{+} & \mathrm{T}<10 \mathrm{~ns} & >100 \mathrm{MHz} \\ \mathrm{NV}^{+} \rightarrow \mathrm{NV}^{-} & \mathrm{T}_{1} \approx 50 \mathrm{~ns}, \mathrm{~T}_{2} \approx 330 \mathrm{~ns} & \approx 1 \mathrm{MHz} \\ \mathrm{NV}^{0} \rightarrow \mathrm{NV}^{+} & \mathrm{T}<10 \mathrm{~ns} & >100 \mathrm{MHz} \\ \mathrm{NV}^{+} \rightarrow \mathrm{NV}^{0} & \mathrm{~T} \approx 100 \mathrm{~ns} & \approx 10 \mathrm{MHz}\end{array}$

measured time constants, a switching frequency between approximately $1 \mathrm{MHz}$ (charging NV) and $100 \mathrm{MHz}$ (discharging NV) seems to be possible. This is much faster than the rate of hyperfine interaction between an electron and nuclear spin of $\mathrm{A}_{/ /}=2.66 \mathrm{kHz}[13]$.

With the help of the Software ATLAS from Silvaco Inc., we simulated this NV charge state switching by the Al-gate of the two-dimensional diamond Schottky diode to get a deeper under- standing of the underlying mechanisms. The NV centre is switched from $\mathrm{NV}^{-} \rightarrow \mathrm{NV}^{0} \rightarrow \mathrm{NV}^{+}$or switched from $\mathrm{NV}^{+} \rightarrow \mathrm{NV}^{0} \rightarrow \mathrm{NV}^{-}$via an interaction with the two-dimensional hole channel, i.e., the centre changes its charge state upon capturing holes from the channel (non-radiative transition, Shockley-Read-Hall mechanism) or thermally emitting holes into the channel. Details of the results of charge state switching simulations and the deduced charge state switching mechanisms will be published in an upcoming paper. 
After several 1000 switching cycles, the measured time constants for discharging $\mathrm{NV}^{-}$increases more than one order of magnitude as shown in Figure 7, indicating a degradation effect of the diode properties.

Since the time constant increases above the time resolution of the measurement, we can now measure two time constants for the process of discharging $\mathrm{NV}^{-} \rightarrow \mathrm{NV}^{+}$like for the process of charging $\mathrm{NV}^{+} \rightarrow \mathrm{NV}^{-}$, too. From this, we can conclude that the transition $\mathrm{NV}^{-} \leftrightarrow \mathrm{NV}^{+}$is a two-step process with $\mathrm{NV}^{0}$ as an intermediate state. This assumption is supported by the result of the simultaneous measurement of $\mathrm{NV}^{-}$and $\mathrm{NV}^{0}$ intensity during switching between $\mathrm{NV}^{-}$and $\mathrm{NV}^{+}$(Figure 8). It shows the occurrence of $\mathrm{NV}^{0}$ emission during the transition between the negative and positive charge state.

The larger time constant of the second step in the two-step process of charging/discharging the NV centre (see Figure $6 \mathrm{~b}$ and Figure 7) can be explained by the change of the charge carrier capture cross section of the NV centre. Generally, it is well known that the charge carrier capture cross section is larger for a charged defect centre (here: $\mathrm{NV}^{-}$or $\mathrm{NV}^{+}$) compared to a neutral defect centre (here: $\mathrm{NV}^{0}$ ).

The observed degradation of NV-switching in Figure 7 and diode properties respectively after many switching cycles is due to the interaction of the H-terminated diamond surface with air, particularly with oxygen $\left(\mathrm{O}_{2}\right)$ and ozone $\left(\mathrm{O}_{3}\right)$ [29]. This interaction is catalysed by illumination of the surface with laser light and application of potential on the diode. Due to this reaction the diamond surface is locally O-terminated leading to an irreversible disappearance of the two-dimensional hole channel. This assumption is supported by the fact that after many thou-

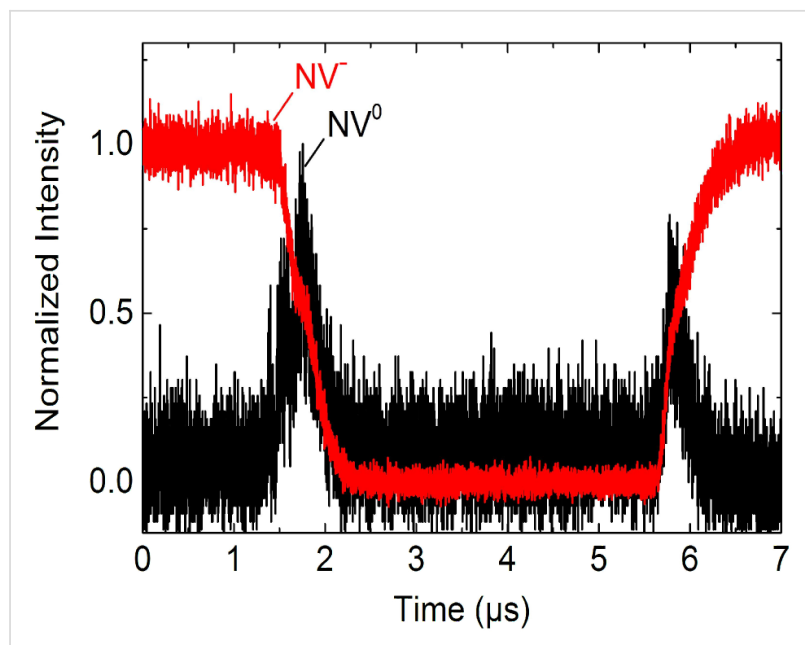

Figure 8: Simultaneous measurement of $\mathrm{NV}^{-}$and $\mathrm{NV}^{0}$ intensity. A time-resolved measurement of $\mathrm{NV}^{-}$(red curve) and $\mathrm{NV}^{0}$ (black curve) intensity simultaneously during switching between $\mathrm{NV}^{-}$and $\mathrm{NV}^{+}$shows that the charging and discharging process is a two-step process with $\mathrm{N} \mathrm{V}^{0}$ as an intermediate state.

sands of switching cycles, it is not possible any more to switch the NV charge state actively. Since the stability of the twodimensional hole channel is influenced by the interaction with the atmosphere, the H-terminated diamond surface need to be passivated to get a stable device operation and NV manipulation. One passivation method was suggested by Hiraiwa et al. by covering the surface with an $\mathrm{Al}_{2} \mathrm{O}_{3}$ film using an atomiclayer-deposition (ALD) method with an $\mathrm{H}_{2} \mathrm{O}$ oxidant at $450{ }^{\circ} \mathrm{C}$ [29]. They could show that this film does not destroy the $\mathrm{C}-\mathrm{H}$ bonds as well as the atmospheric adsorbate layer on top of the $\mathrm{H}$-terminated surface which is required for the generation of a two-dimensional hole channel. In this way, the hole channel and thus the diode properties are preserved to temperatures up to (a)

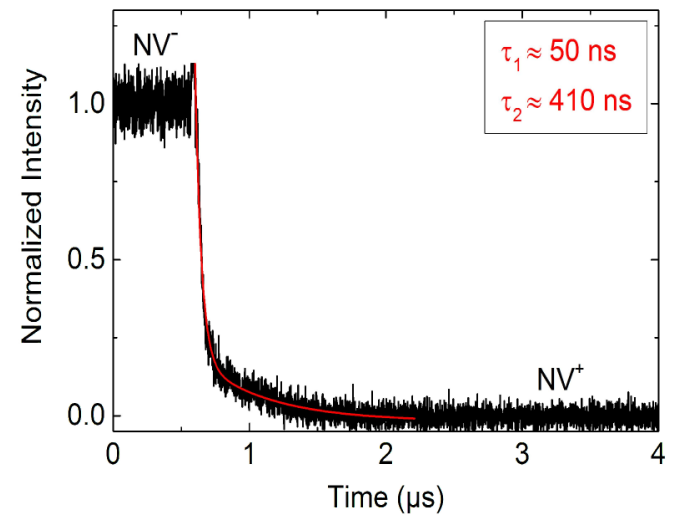

(b)

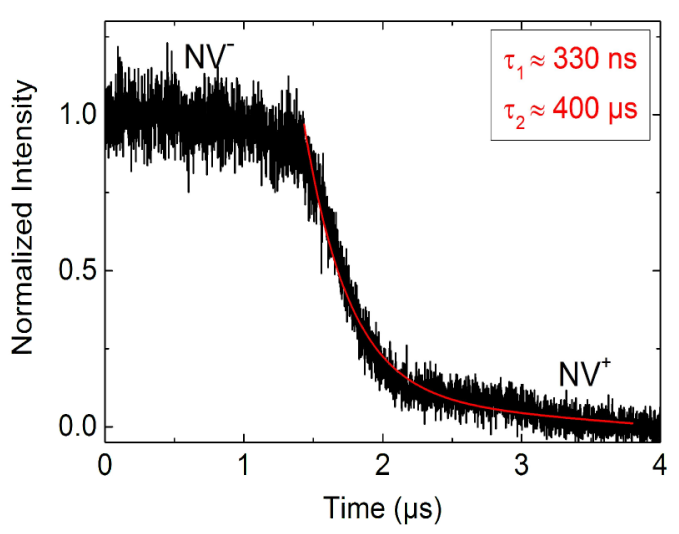

Figure 7: Degradation effect. After several 1000 switching cycles, the measured time constants for discharging $\mathrm{NV}^{-}$increases to (a) approximately $500 \mathrm{~ns}$. After additional several 1000 switching cycles the measured time constant (b) increases by one order of magnitude to approximately $500 \mu \mathrm{s}$. This indicates a degradation effect of the diode properties. The reasons for the observed degradation is explained in the text. Since the time constant increases above the time resolution of the measurement setup, we can now measure two time constants for the process of discharging $\mathrm{NV}^{-}$to $\mathrm{NV}^{+}$, indicating a two-step process. 
$550{ }^{\circ} \mathrm{C}$ in air. Chemically, this stability is supported by the fact that both the thermal decomposition of $\mathrm{C}-\mathrm{H}$ bonds and the reaction between $\mathrm{C}-\mathrm{H}$ bonds and $\mathrm{H}_{2} \mathrm{O}$ in the $\mathrm{Al}_{2} \mathrm{O}_{3}$ layer are endothermic processes. This passivation method for preserving the properties of the two-dimensional hole channel and thus of the two-dimensional Al-Schottky diode for active manipulation of single NV centres will be evaluated in the upcoming experiments.

\section{Summary and Conclusion}

In this paper, we presented the experimental results of active control and high-frequency switching of the charge state of single and near-surface NV centres with a two-dimensional Al-Schottky diode from diamond. By applying potentials on the Al-Schottky contact and simultaneous illumination of an NV centre with a $520 \mathrm{~nm} \mathrm{cw}$ laser light, we can actively switch single $\mathrm{NV}$ centres between the charge states $\mathrm{NV}^{+}, \mathrm{NV}^{0}$ and $\mathrm{NV}^{-}$. This is due to the field-induced band bending modulation in the depletion region of the Schottky junction which shifts the Fermi-level over NV charge transition levels in such a way that the charge state of a single NV centre and thus its electrical and optical properties are tuned.

The measured switching frequency for the transition $\mathrm{NV}^{-} / \mathrm{NV}^{0}$ $\rightarrow \mathrm{NV}^{+}$and $\mathrm{NV}^{+} \rightarrow \mathrm{NV}^{0} / \mathrm{NV}^{-}$is $100 \mathrm{MHz}$ and $1 \mathrm{MHz}$ respectively. The frequencies of combined optical and electrical switching are three orders of magnitude higher than the rate of hyperfine interaction between an electron and nuclear spin of $\mathrm{A}_{/ /}=2.66 \mathrm{kHz}$. Furthermore, they are higher compared to the frequencies achieved by other technologies. For example, for pure optically induced switching between $\mathrm{NV}^{0}$ and $\mathrm{NV}^{-}$, the maximum rate is $1 \mathrm{MHz}$, due to two-photon-absorption processes [30,31]. And for a pure electrically induced switching between $\mathrm{NV}^{0}$ and $\mathrm{NV}^{-}$in the intrinsic layer of a diamond pin diode, the maximum rate is $0.7 \mathrm{MHz}[13,30]$, due to a larger capacitance of the three-dimensional diode. In addition - contrary to the above mentioned alternative technologies - with a twodimensional Al-Schottky diode, presented in this paper, it is possible to switch between all three charge states $\mathrm{NV}^{+}, \mathrm{NV}^{0}$ and $\mathrm{NV}^{-}$, since it enables a shift of the Fermi-level over both $\mathrm{NV}$ charge transition levels $\mathrm{NV}^{+/ 0}$ and $\mathrm{NV}^{0 /-}$. From simulations we could deduce that holes from the two-dimensional channel play a dominant role in switching the NV charge state, i.e., the centre changes its charge state upon capturing holes from the channel (non-radiative transition, Shockley-Read-Hall mechanism) or thermally emitting holes into the channel, depending on the direction of the bias switching.

This high-frequency charge state switching via a planar diode structure would open the door for many quantum optical applications such as a quantum registers with single NVs for initial- ization, processing and read-out as well as single ${ }^{13} \mathrm{C}$ atoms for storage of quantum information. This could be realised with a suitable patterning of the Al-gates, such that single NV centres could be addressed individually. Furthermore, a control of the spectral emission properties of NVs as a single photon emitters - embedded in photonic structures for example - could be realized which would be vital for quantum communication and cryptography.

\section{Acknowledgements}

The authors gratefully acknowledge financial support by Baden-Württemberg Stiftung in the framework of the part project D5 of Kompetenznetz Funktionelle Nanostrukturen (KFN IV).

\section{References}

1. Kurtsiefer, C.; Mayer, S.; Zarda, P.; Weinfurter, H. Phys. Rev. Lett. 2000, 85, 290-293. doi:10.1103/PhysRevLett.85.290

2. Jelezko, F.; Wrachtrup, J. J. Phys.: Condens. Matter 2004, 16, R1089-R1104. doi:10.1088/0953-8984/16/30/R03

3. Jelezko, F.; Wrachtrup, J. Phys. Status Solidi A 2006, 203, 3207-3225. doi:10.1002/pssa.200671403

4. Maze, J. R.; Stanwix, P. L.; Hodges, J. S.; Hong, S.; Taylor, J. M.; Cappellaro, P.; Jiang, L.; Dutt, M. V. G.; Togan, E.; Zibrov, A. S.; Yacoby, A.; Walsworth, R. L.; Lukin, M. D. Nature 2008, 455, 644-647. doi:10.1038/nature07279

5. Vaijayanthimala, V.; Chang, H.-C. Nanomedicine (London, U. K.) 2009, 4, 47-55. doi:10.2217/17435889.4.1.47

6. Gisin, N.; Ribordy, G.; Tittel, W.; Zbinden, H. Rev. Mod. Phys. 2002, 74, 145-195. doi:10.1103/RevModPhys.74.145

7. Beveratos, A.; Brouri, R.; Gacoin, T.; Villing, A.; Poizat, J.-P.; Grangier, P. Phys. Rev. Lett. 2002, 89, 187901. doi:10.1103/PhysRevLett.89.187901

8. Rondin, L.; Dantelle, G.; Slablab, A.; Grosshans, F.; Treussart, F.; Bergonzo, P.; Perruchas, S.; Gacoin, T.; Chaigneau, M.; Chang, H.-C.; Jacques, V.; Roch, J.-F. Phys. Rev. B: Condens. Matter Mater. Phys. 2010, 82, 115449. doi:10.1103/PhysRevB.82.115449

9. Fu, K.-M. C.; Santori, C.; Barclay, P. E.; Beausoleil, R. G. Appl. Phys. Lett. 2010, 96, 121907. doi:10.1063/1.3364135

10. Hauf, M. V.; Grotz, B.; Naydenov, B.; Dankerl, M.; Pezzagna, S.; Meijer, J.; Jelezko, F.; Wrachtrup, J.; Stutzmann, M.; Reinhard, F.; Garrido, J. A. Phys. Rev. B: Condens. Matter Mater. Phys. 2011, 83, 081304. doi:10.1103/PhysRevB.83.081304

11. Maurer, P. C.; Kucsko, G.; Latta, C.; Jiang, L.; Yao, N. Y.; Bennett, S. D.; Pastawski, F.; Hunger, D.; Chisholm, N.; Markham, M.; Twitchen, D. J.; Cirac, J. I.; Lukin, M. D. Science 2012, 336, 1283-1286. doi:10.1126/science.1220513

12. Dolde, F.; Bergholm, V.; Wang, Y.; Jakobi, I.; Naydenov, B.; Pezzagna, S.; Meijer, J.; Jelezko, F.; Neumann, P.; Schulte-Herbrüggen, T.; Biamonte, J.; Wrachtrup, J. Nat. Commun. 2014, 5, 3371. doi:10.1038/ncomms4371

13. Doi, Y.; Makino, T.; Kato, H.; Takeuchi, D.; Ogura, M.; Okushi, H.; Morishita, H.; Tashima, T.; Miwa, S.; Yamasaki, S.; Neumann, P.; Wrachtrup, J.; Suzuki, Y.; Mizuochi, N. Phys. Rev. X 2014, 4, 011057. doi:10.1103/PhysRevX.4.011057

14. Cui, S.; Hu, E. L. Appl. Phys. Lett. 2013, 103, 051603. doi:10.1063/1.4817651 
15. Grotz, B.; Hauf, M. V.; Dankerl, M.; Naydenov, B.; Pezzagna, S.; Meijer, J.; Jelezko, F.; Wrachtrup, J.; Stutzmann, M.; Reinhard, F.; Garrido, J. A. Nat. Commun. 2012, 3, 729. doi:10.1038/ncomms1729

16. Kato, H.; Wolfer, M.; Schreyvogel, C.; Kunzer, M.; Müller-Sebert, W.; Obloh, H.; Yamasaki, S.; Nebel, C. Appl. Phys. Lett. 2013, 102, 151101. doi:10.1063/1.4801871

17. Hauf, M. V.; Simon, P.; Aslam, N.; Pfender, M.; Neumann, P.; Pezzagna, S.; Meijer, J.; Wrachtrup, J.; Stutzmann, M.; Reinhard, F.; Garrido, J. A. Nano Lett. 2014, 14, 2359-2364. doi:10.1021/nl4047619

18. Nebel, C. E.; Rezek, B.; Shin, D.; Watanabe, H. Phys. Status Solidi A 2006, 203, 3273-3298. doi:10.1002/pssa.200671401

19. Garrido, J. A.; Nebel, C. E.; Stutzmann, M.; Snidero, E.; Bergonzo, P. Appl. Phys. Lett. 2002, 81, 637-639. doi:10.1063/1.1496495

20. Schreyvogel, C.; Wolfer, M.; Kato, H.; Schreck, M.; Nebel, C. E. Sci. Rep. 2014, 4, 3634. doi:10.1038/srep03634

21.Schreyvogel, C.; Polyakov, V.; Wunderlich, R.; Meijer, J.; Nebel, C. E. Sci. Rep. 2015, 5, 12160. doi:10.1038/srep12160

22. Füner, M.; Wild, C.; Koidl, P. Appl. Phys. Lett. 1998, 72, 1149-1151. doi:10.1063/1.120997

23. Thomas, E. L. H.; Nelson, G. W.; Mandal, S.; Foord, J. S.; Williams, O. A. Carbon 2014, 68, 473-479. doi:10.1016/j.carbon.2013.11.023

24. Ziegler, J. F.; Ziegler, M. D.; Biersack, J. P. Nucl. Instrum. Methods Phys. Res., Sect. B 2010, 268, 1818-1823. doi:10.1016/j.nimb.2010.02.091

25. Acosta, V. M.; Bauch, E.; Ledbetter, M. P.; Santori, C.; Fu, K.-M. C.; Barclay, P. E.; Beausoleil, R. G.; Linget, H.; Roch, J. F.; Treussart, F.; Chemerisov, S.; Gawlik, W.; Budker, D. Phys. Rev. B 2009, 80, 115202. doi:10.1103/PhysRevB.80.115202

26. Tallaire, A.; Collins, A. T.; Charles, D.; Achard, J.; Sussmann, R.; Gicquel, A.; Newton, M. E.; Edmonds, A. M.; Cruddace, R. J. Diamond Relat. Mater. 2006, 15, 1700-1707. doi:10.1016/j.diamond.2006.02.005

27. Beveratos, A.; Kühn, S.; Brouri, R.; Gacoin, T.; Poizat, J.-P.; Grangier, P. Eur. Phys. J. D 2002, 18, 191-196. doi:10.1140/epjd/e20020023

28. Brouri, R.; Beveratos, A.; Poizat, J.-P.; Grangier, P. Opt. Lett. 2000, 25, 1294-1296. doi:10.1364/OL.25.001294

29. Hiraiwa, A.; Daicho, A.; Kurihara, S.; Yokoyama, Y.; Kawarada, H. J. Appl. Phys. 2012, 112, 124504. doi:10.1063/1.4769404

30. Waldherr, G.; Beck, J.; Steiner, M.; Neumann, P.; Gali, A.; Frauenheim, T.; Jelezko, F.; Wrachtrup, J. Phys. Rev. Lett. 2011, 106, 157601. doi:10.1103/PhysRevLett.106.157601

31. Aslam, N.; Waldherr, G.; Neumann, P.; Jelezko, F.; Wrachtrup, J. New J. Phys. 2013, 15, 013064. doi:10.1088/1367-2630/15/1/013064

\section{License and Terms}

This is an Open Access article under the terms of the Creative Commons Attribution License (http://creativecommons.org/licenses/by/4.0), which permits unrestricted use, distribution, and reproduction in any medium, provided the original work is properly cited.

The license is subject to the Beilstein Journal of Nanotechnology terms and conditions: (http://www.beilstein-journals.org/bjnano)

The definitive version of this article is the electronic one which can be found at:

doi:10.3762/bjnano.7.165 